PROCEEDINGS OF THE

AMERICAN MATHEMATICAL SOCIETY

Volume 134, Number 10, October 2006, Pages 3015-3024

S $0002-9939(06) 08357-2$

Article electronically published on May 8, 2006

\title{
THE REAL RANK ZERO PROPERTY OF CROSSED PRODUCT
}

\author{
XIAOCHUN FANG
}

(Communicated by David R. Larson)

\begin{abstract}
Let $A$ be a unital $C^{*}$-algebra, and let $(A, G, \alpha)$ be a $C^{*}$-dynamical system with $G$ abelian and discrete. In this paper, we introduce the continuous affine map $R$ from the trace state space $T\left(A \times{ }_{\alpha} G\right)$ of the crossed product $A \times{ }_{\alpha} G$ to the $\alpha$-invariant trace state space $T(A)_{\alpha^{*}}$ of $A$. If $A \times_{\alpha} G$ is of real rank zero and $\hat{G}$ is connected, we have proved that $R$ is homeomorphic. Conversely, if $R$ is homeomorphic, we also get some properties and real rank zero characterization of $A \times_{\alpha} G$. In particular, in that case, $A \times{ }_{\alpha} G$ is of real rank zero if and only if each unitary element in $A \times_{\alpha} G$ with the form $u_{A} \prod_{i=1}^{n} x_{i}^{*} y_{i}^{*} x_{i} y_{i}$ can be approximated by the unitary elements in $A \times_{\alpha} G$ with finite spectrum, where $u_{A} \in U_{0}(A), x_{i}, y_{i} \in C_{c}(G, A) \cap U_{0}\left(A \times_{\alpha} G\right)$, and if moreover $A$ is a unital inductive limit of the direct sums of non-elementary simple $C^{*}$-algebras of real rank zero, then the $u_{A}$ above can be cancelled.
\end{abstract}

\section{INTRODUCTION}

Let $A$ be a unital $C^{*}$-algebra, and let $A_{s a}$ be the set consists of all self-adjoint elements in $A$. The real rank of $A$ is the smallest integer, $R R(A)$, such that for each $n$-tuple $\left(x_{1}, x_{2}, \cdots, x_{n}\right)$ of elements in $A_{s a}$ with $n \leq R R(A)+1$, and every $\varepsilon>0$, there is an $n$-tuple $\left(y_{1}, y_{2}, \cdots, y_{n}\right)$ of elements in $A_{s a}$ such that $\sum_{k=1}^{n} y_{k}^{*} y_{k}$ is invertible and $\left\|\sum_{k=1}^{n}\left(x_{k}-y_{k}\right)^{2}\right\|<\varepsilon$. In particular, $R R(A)=0$ if and only if the invertible self-adjoint elements are dense in $A_{s a}$, which is equivalent to the fact that the self-adjoint elements with finite spectrum are dense in $A_{s a}$. For a non-unital $C^{*}$-algebra $A$, the real rank of $A$ is $R R(\tilde{A})$, where $\tilde{A}$ is the unitization of $A$. The real rank zero property has been widely studied by many authors, for example L. Brown, H. Lin, G. K. Pedersen, S. Zhang and so on. Let $A$ be a $C^{*}$-algebra, let $G$ be a locally compact Hausdorff group, and let $(A, G, \alpha)$ be a $C^{*}$ dynamical system. There are also many efforts that have been made to understand the relation between the real ranks of $A$ and of $A \times{ }_{\alpha} G$, in particular, between the real rank zero properties of $A$ and $A \times{ }_{\alpha} G$. For example, in 4, it was guessed that $R R\left(A \times_{\alpha} G\right) \leq \operatorname{dim} \hat{G}+R R(A)$ for $G$ finite. In [3], by using the Rokhlin property, some statements equivalent to $R R\left(A \times{ }_{\alpha} \mathbf{Z}\right)=0$ were given for $A=M_{2^{\infty}}$, which were generalized later in 9 for $A$ to be a UHF algebra. For a $C^{*}$-algebra $A$, a bounded functional $\tau$ of $A$ is called a trace state, if $\lim _{\lambda} \tau\left(e_{\lambda}\right)=1$, where

Received by the editors January 3, 2005 and, in revised form, May 9, 2005.

2000 Mathematics Subject Classification. Primary 46L05; Secondary 46L35, 46L40.

Key words and phrases. Real rank zero, crossed product, trace state space.

This article was supported by the National Natural Science Foundation of China (10271090).

(c)2006 American Mathematical Society Reverts to public domain 28 years from publication 3015 
$\left\{e_{\lambda}\right\}$ is one approximate unit of $A$, and $\tau(a b)=\tau(b a)$ for any $a, b \in A$. The trace state space of $A$, which is notated by $T(A)$, is the set consisting of all trace states of $A$. For a $C^{*}$-dynamical system $(A, G, \alpha), \alpha$ induces a canonical action $\alpha^{*}$ on $T(A):\left(\alpha_{t}^{*}(\tau)\right)(a)=\tau\left(\alpha_{t}(a)\right)(\forall a \in A, \tau \in T(A), t \in G)$. A trace state $\tau \in T(A)$ is called $\alpha$-invariant if $\alpha_{t}^{*}(\tau)=\tau(\forall t \in G)$. Let $T(A)_{\alpha^{*}}$ be the set consisting of all $\alpha$-invariant trace states. If moreover $G$ is discrete and $A$ is unital, then $A$ can be viewed as a unital subalgebra of $A \times{ }_{\alpha} G$. Therefore for any trace state of $A \times{ }_{\alpha} G$, we get a trace state of $A$ by restriction, which is moreover $\alpha$-invariant by direct computation, i.e. there is a canonical mapping $R: T\left(A \times_{\alpha} G\right) \rightarrow T(A)_{\alpha^{*}}$. Since the trace state space is one of Elliott's classification invariants for unital amenable $C^{*}$-algebras, we want to find how much the mapping $R$ reflects the relation of the real rank zero properties of $A$ and $A \times{ }_{\alpha} G$. In this paper, for $G$ abelian, if $A \times{ }_{\alpha} G$ is of real rank zero, and $\hat{G}$ is connected, we prove that $R$ is homeomorphic. Conversely, if $R$ is homeomorphic, we will get some properties and real rank zero characterization of $A \times_{\alpha} G$. In particular, in that case, $A \times{ }_{\alpha} G$ is of real rank zero if and only if each unitary element in $A \times_{\alpha} G$ with the form $u_{A} \prod_{i=1}^{n} x_{i}^{*} y_{i}^{*} x_{i} y_{i}$ can be approximated by the unitary elements in $A \times{ }_{\alpha} G$ with finite spectrum, where $u_{A} \in U_{0}(A), x_{i}, y_{i} \in C_{c}(G, A) \cap U_{0}\left(A \times_{\alpha} G\right)$, and if moreover $A$ is a unital inductive limit of the direct sums of non-elementary simple $C^{*}$-algebras of real rank zero, then $A \times{ }_{\alpha} G$ is of real rank zero if and only if each unitary element in $A \times{ }_{\alpha} G$ with

the form $\prod_{i=1}^{n} x_{i}^{*} y_{i}^{*} x_{i} y_{i}$ can be approximated by the unitary elements in $A \times{ }_{\alpha} G$ with finite spectrum.

\section{Main Results}

We should first recall some basic notations and definitions in $K$-theory which will play roles later. Let $A$ be a unital $C^{*}$-algebra. For each integer $k$, we denote the unitary group of $M_{k}(A)$ by $U^{k}(A)$, and the subgroup of $U^{k}(A)$ consisting of all elements connected to the unit of $M_{k}(A)$ by $U_{0}^{k}(A)$. Viewing $U^{k}(A)$ $\left(U_{0}^{k}(A)\right)$ as a subgroup of $U^{k+1}(A)\left(U_{0}^{k+1}(A)\right)$ by identifying $\operatorname{diag}(u, 1)$ with $u$ for any $u \in U^{k}(A)\left(U_{0}^{k}(A)\right)$, we let $U^{\infty}(A)=\lim _{k \rightarrow \infty} U^{k}(A)$ as a topological group with the inductive limit topology coming from the inclusion $U^{k}(A) \subseteq U^{k+1}(A)$, and similarly let $U_{0}^{\infty}(A)=\lim _{k \rightarrow \infty} U_{0}^{k}(A)$ as a topological group with the inductive limit topology coming from the inclusion $U_{0}^{k}(A) \subseteq U_{0}^{k+1}(A)$. For any $n \in \mathbf{N} \cup\{\infty\}$, we denote the commutator subgroup of $U^{n}(A)$ and $U_{0}^{n}(A)$ by $D U^{n}(A)$ and $D U_{0}^{n}(A)$ respectively, and let $\overline{D U^{n}(A)}\left(\overline{D U_{0}^{n}(A)}\right)$ be the closure of $D U^{n}(A)\left(D U_{0}^{n}(A)\right)$. For any fixed $n \in \mathbf{N} \cup\{\infty\}$, we let $q^{0}$ be the quotient map from $U_{0}^{n}(A)$ to $U_{0}^{n}(A) / \overline{D U_{0}^{n}(A)}$.

Let $T(A)$ be the trace state space of $A$, which is a compact Hausdorff space with the $w^{*}$ topology. (In this paper, we always assume that $T(A) \neq \emptyset$.) Let Af $f T(A)$ denote the space of continuous affine real-valued function on $T(A)$, which is a real Banach space with the standard function norm. Let $\eta:[0,1] \mapsto U_{0}^{n}(A)$ $(n \in \mathbf{N} \cup\{\infty\})$ be a piecewise smooth path of unitary from 1 ; we define $\Delta_{n}^{1}(\eta) \in$ $\operatorname{AffT}(A)$ by

$$
\Delta_{n}^{1}(\eta)(\omega)=\frac{1}{2 \pi i} \int_{0}^{1} \omega\left(\eta^{\prime}(t) \eta(t)^{*}\right) d t, \omega \in T(A)
$$


The key observations (see [1], Lemma 3) are:

(1) $\Delta_{n}^{1}(\eta)$ depends only on $\eta$ up to homotopy with fixed endpoints, and

(2) $\Delta_{n}^{1}\left(\eta_{1} \eta_{2}\right)=\Delta_{n}^{1}\left(\eta_{1}\right)+\Delta_{n}^{1}\left(\eta_{2}\right)$ for any piecewise smooth paths $\eta_{i}$ with $\eta_{i}(1)=1$ $(i=1,2)$.

It follows that $\Delta_{n}^{1}$ defines a group homomorphism $\Delta_{n}^{0}: \pi_{1}\left(U_{0}^{n}(A)\right) \rightarrow \operatorname{AffT}(A)$, where $\pi_{1}\left(U_{0}^{n}(A)\right)$ is the fundamental group of $U_{0}^{n}(A)$. For $n=\infty, \pi_{1}\left(U_{0}^{\infty}(A)\right)=$ $K_{0}(A)$ by Bott periodicity, and $\Delta_{\infty}^{0}$ is just the well-known canonical map $\rho$ : $K_{0}(A) \rightarrow \operatorname{AffT}(A)$. Let $q$ be the quotient map from $\operatorname{AffT}(A)$ to $\operatorname{AffT}(A) /$ $\overline{\Delta_{n}^{0}\left(\pi_{1}\left(U_{0}^{n}(A)\right)\right)}$. Since for any unital $C^{*}$-algebra $B, U_{0}(B)$ is generated algebraically by $\left\{e^{i x}: x \in B_{s a}\right\}$, for each unitary $u \in U_{0}^{n}(A)$, there is a piecewise smooth path $\eta_{u}$ in $U_{0}^{n}(A)$ from 1 to $u$. Therefore for each $n \in \mathbf{N} \cup\{\infty\}$, we can define a group homomorphism:

$$
\Delta_{n}: U_{0}^{n}(A) \mapsto A f f T(A) \overline{\Delta_{n}^{0}\left(\pi_{1}\left(U_{0}^{n}(A)\right)\right)}, \quad \Delta_{n}(u)=q\left(\Delta_{n}^{1}\left(\eta_{u}\right)\right) .
$$

This homomorphism makes it possible to get the information of the real rank zero property of $A \times{ }_{\alpha} G$, which is relative to $U_{0}\left(A \times{ }_{\alpha} G\right)$, from the mapping $R$ : $T\left(A \times_{\alpha} G\right) \rightarrow T(A)_{\alpha^{*}}$.

Lemma 1. Let $(A, G, \alpha)$ be a $C^{*}$-dynamical system with $G$ abelian and discrete. There is a canonical faithful expectation $\phi$ of $A \times_{\alpha} G$ onto $A$ with $\phi(f)=f(0)$, where $f \in C_{c}(G, A)$ and 0 is the unit of $G$.

Proof. By duality, we have a $C^{*}$-dynamical system $\left(A \times{ }_{\alpha} G, \hat{G}, \hat{\alpha}\right)$, where $\hat{\alpha}_{\sigma}(f)(t)=$ $(t, \sigma) f(t)$ for every $f \in C_{c}(G, A), t \in G, \sigma \in \hat{G}$. Then let $\phi: A \times_{\alpha} G \rightarrow A$ be $\phi(x)=\int_{\hat{G}} \hat{\alpha}_{\sigma}(x) \mathrm{d} \mu(\sigma)\left(\forall x \in A \times_{\alpha} G\right)$, where $\mu$ is the canonical Haar probability on the compact group $\hat{G}$. Since $G$ is discrete, $A \subseteq A \times{ }_{\alpha} G$, and $A=\{x \in$ $A \times_{\alpha} G: \hat{\alpha}_{\sigma}(x)=x$ for $\left.\left.\forall \sigma \in \hat{G}\right)\right\}$. So the image of $\phi$ is included in $A$, and for $\forall a, b \in A, x \in A \times{ }_{\alpha} G$,

$$
\phi(a x b)=\int_{\hat{G}} \hat{\alpha}_{\sigma}(a x b) \mathrm{d} \mu(\sigma)=a \phi(x) b, \phi(a)=\int_{\hat{G}} \hat{\alpha}_{\sigma}(a) \mathrm{d} \mu(\sigma)=a .
$$

It is easy to see that $\phi(f)=f(0)$ for $f \in C_{c}(G, A)$.

By the definition of $\phi, \phi$ is a faithful completely positive map, and so a faithful expectation from $A \times{ }_{\alpha} G$ onto $A$.

Lemma 2. Let $(A, G, \alpha)$ be a $C^{*}$-dynamical system with $G$ abelian and discrete and $A$ unital, and let $T(A)_{\alpha_{*}}=\left\{\tau \in T(A): \tau \circ \alpha_{t}=\tau, \forall t \in G\right\}$. Then there are continuous affine maps $\Phi: T(A)_{\alpha_{*}} \rightarrow T\left(A \times_{\alpha} G\right)$ and $R: T\left(A \times_{\alpha} G\right) \rightarrow T(A)_{\alpha_{*}}$ such that $R \circ \Phi=i d$ and the image of $\Phi$ is

$$
T\left(A \times_{\alpha} G\right)_{\hat{\alpha}_{*}}=\left\{\tau \in T\left(A \times_{\alpha} G\right): \tau \circ \hat{\alpha}_{\sigma}=\tau, \forall \sigma \in \hat{G}\right\} .
$$

Therefore $\Phi$ is an affine homeomorphism from $T(A)_{\alpha_{*}}$ onto $T\left(A \times_{\alpha} G\right)_{\hat{\alpha}_{*}}$.

Proof. Let $i$ be the canonical inclusion of $A$ in $A \times{ }_{\alpha} G$, and $R(\omega)=\omega \circ i$ for each $\omega \in T\left(A \times_{\alpha} G\right)$. It is easy to see that $R(\omega) \in T(A)_{\alpha_{*}}$. By Lemma 1 , let $\phi$ be the canonical faithful expectation from $A \times{ }_{\alpha} G$ onto $A$, and let $\Phi(\tau)=\tau \circ \phi$ for each $\tau \in T(A)_{\alpha_{*}}$.

For each $f, g \in C_{c}(G, A) \subseteq A \times{ }_{\alpha} G$, since

$$
\phi(f * g)=(f * g)(0)=\int f(s) \alpha_{s}(g(-s)) \mathrm{d} s,
$$


it follows that

$$
\begin{aligned}
\Phi(\tau)(f * g) & =\tau \circ \phi(f * g)=\int \tau\left(f(s) \alpha_{s}(g(-s))\right) \mathrm{d} s \\
& =\int \tau\left(\alpha_{s}\left(\alpha_{-s}(f(s)) g(-s)\right)\right) \mathrm{d} s \\
& =\int \tau\left(\alpha_{-s}(f(s)) g(-s)\right) \mathrm{d} s \\
& =\int \tau\left(g(-s) \alpha_{-s}(f(s))\right) \mathrm{d} s \\
& =\int \tau\left(g(s) \alpha_{s}(f(-s))\right) \mathrm{d} s \\
& =\tau \circ \phi(g * f)=\Phi(\tau)(g * f) .
\end{aligned}
$$

So $\Phi(\tau) \in T\left(A \times_{\alpha} G\right)$, and it is easy to see $R \circ \Phi(\tau)=\tau\left(\forall \tau \in T(A)_{\alpha_{*}}\right)$. Since for each $\sigma_{0} \in \hat{G}, \Phi(\tau) \circ \hat{\alpha}_{\sigma_{0}}=\tau \circ \phi \circ \hat{\alpha}_{\sigma_{0}}$ and $\phi \circ \hat{\alpha}_{\sigma_{0}}(x)=\int_{\hat{G}} \hat{\alpha}_{\sigma} \hat{\alpha}_{\sigma_{0}}(x) \mathrm{d} \sigma=\phi(x)$ $\left(\forall x \in A \times{ }_{\alpha} G\right)$, it follows that $\Phi(\tau) \circ \hat{\alpha}_{\sigma}=\Phi(\tau)(\forall \sigma \in \hat{G})$, i.e. $\Phi(\tau) \in T\left(A \times_{\alpha} G\right)_{\hat{\alpha}_{*}}$.

If $\omega \in T\left(A \times_{\alpha} G\right)$ with $\omega \circ \hat{\alpha}_{\sigma}=\omega(\forall \sigma \in \hat{G})$, then $\Phi(R(\omega))=\omega \circ i \circ \phi$. For any $t \in G, a \in A$, let $a \delta(t, \cdot)$ be the element in $C_{c}(G, A)$ with value $a$ at $t, 0$ at any other $s \in G$ which is not $t$, then for every $f \in C_{c}(G, A), \omega \circ i \circ \phi(f)=\omega(f(0) \delta(0, \cdot))$. Since for any $\sigma \in \hat{G}$,

$$
\omega(a \delta(t, \cdot))=\omega\left(\hat{\alpha}_{\sigma}(a \delta(t, \cdot))\right)=(t, \sigma) \omega(a \delta(t, \cdot)),
$$

$\omega(a \delta(t, \cdot))=0$, if $t \neq 0$, and so $\omega(f(\cdot))=\omega(f(0) \delta(0, \cdot))=\omega \circ i \circ \phi(f(\cdot))$.

Therefore $\omega=\omega \circ i \circ \phi=\Phi(R(\omega)) \in$ image of $\Phi$, i.e. the image of $\Phi$ is $\{\tau \in$ $T\left(A \times{ }_{\alpha} G\right): \tau \circ \hat{\alpha_{\sigma}}=\tau$ for $\left.\forall \sigma \in \hat{G}\right\}$.

Proposition 1. Let $(B, H, \beta)$ be a $C^{*}$-dynamical system with $H$ connected and $B$ of real rank zero. Then for any $\tau \in T(B), h \in H, \tau \circ \beta_{h}=\tau$, therefore $T(B)_{\beta_{*}}=T(B)$.

Proof. First we assume that $B$ is unital, let $\omega \in T(B)$, and let $p$ be a projection in $B$. Then the mapping $h \rightarrow \omega\left(\beta_{h}(p)\right), \forall h \in H$, is continuous on $H$. For any $h_{0} \in H$, there is a neighborhood $V_{0}$ of $h_{0}$ such that $\left\|\beta_{h}(p)-p\right\|<1$ for any $h \in V_{0}$, and so there is a unitary element $u_{h}$ in $B$ such that $\beta_{h}(p)=u_{h} p u_{h}^{*}$. Since $\omega$ is a trace, the mapping $h \rightarrow \omega\left(\beta_{h}(p)\right)$ is locally constant on $H$. Let $U=\left\{h \in H \mid \omega\left(\beta_{h}(p)\right)=\omega(p)\right\}$; then, by the discussion above $U$ is a non-empty clopen set. Therefore $U=H$ by the connectedness of $H$, i.e. for any $\omega \in T(B)$, projection $p \in B, \omega\left(\beta_{h}(p)\right)=\omega(p)(\forall h \in H)$. Since $B$ is real rank zero, for any $b \in B, b$ can be approximated by the linear combination of projections in $B$, and so $\omega\left(\beta_{h}(b)\right)=\omega(b)(\forall h \in H)$. This completes the proof in the unital case.

Now if $B$ is non-unital, and we let $\widetilde{B}=B+\mathbf{C} 1$ be the unitization of $B$, then $\widetilde{B}$ is of real rank zero by definition. For any $h \in H$, let $\widetilde{\beta_{h}}$ be the automorphism of $\widetilde{B}$ such that $\widetilde{\beta_{h}}(b+\gamma 1)=\beta_{h}(b)+\gamma 1$. Then $\widetilde{\beta}: H \rightarrow A u t(\widetilde{B}), h \rightarrow \widetilde{\beta_{h}}$, define a $C^{*}$-dynamical system $(\widetilde{B}, H, \widetilde{\beta})$. Therefore $T(\widetilde{B})_{\widetilde{\beta}_{*}}=T(\widetilde{B})$. For any $\tau \in T(B)$ (the trace state of $B$ ), we define $\widetilde{\tau}$ by $\widetilde{\tau}(b+\gamma 1)=\tau(b)+\gamma$. It is easy to see $\widetilde{\tau} \in T(\widetilde{B})$ with $\left.\widetilde{\tau}\right|_{B}=\tau$. So $\tau \circ \beta_{h}=\left(\left.\widetilde{\tau}\right|_{B}\right) \circ \beta_{h}=\left.\left(\widetilde{\tau} \circ \widetilde{\beta}_{h}\right)\right|_{B}=\left.\widetilde{\tau}\right|_{B}=\tau$, and so $T(B)_{\beta_{*}}=T(B)$. 
Theorem 1. Let $(A, G, \alpha)$ be a $C^{*}$-dynamical system, with $A$ unital, $G$ abelian and discrete, $\hat{G}$ connected. If $A \times{ }_{\alpha} G$ is of real rank zero, then $T\left(A \times{ }_{\alpha} G\right)=T\left(A \times{ }_{\alpha} G\right)_{\hat{\alpha}_{*}}$, and the mapping $R: T\left(A \times_{\alpha} G\right) \rightarrow T(A)_{\alpha_{*}}$ defined in Lemma 2 is an affine homeomorphism with $R^{-1}=\Phi$.

Proof. Let $\left(A \times{ }_{\alpha} G, \hat{G}, \hat{\alpha}\right)$ be the dual $C^{*}$-dynamical system of $(A, G, \alpha)$. Then $T\left(A \times{ }_{\alpha} G\right)=T\left(A \times_{\alpha} G\right)_{\hat{\alpha}_{*}}$ by Proposition 1. So, keeping the notations as in Lemma $2, \Phi$ is an affine continuous bijection with $\Phi^{-1}=R$. This completes the proof of the theorem.

Note. Conversely, for an abelian group $G$ and a $C^{*}$-dynamical system $(A, G, \alpha)$ with $R: T\left(A \times{ }_{\alpha} G\right) \rightarrow T(A)_{\alpha_{*}}$ homemorphic, we may not get that $A \times_{\alpha} G$ is of real rank zero. The simplest trivial example is that $G=\{e\}$ and $A$ is not of real rank zero. The following is another example for $G$ non-trivial.

Example 1. Let $G$ be a finite abelian group with unit $e$, let $B$ be a unital $C^{*}$ algebra acting on a Hilbert space $\mathbf{H}$, let $A=C(G, B)=C(G) \otimes B$, and let $(A, G, \alpha)$ be a $C^{*}$-dynamical system with $\left(\alpha_{s} y\right)(t)=y(t-s), \forall s, t \in G, y \in A$. Then $A \times{ }_{\alpha} G=C(G, B) \times{ }_{\alpha} G$. Let $\pi: B \rightarrow \mathbf{B}\left(l^{2}(G, \mathbf{H})\right),(\pi(b) \xi)(t)=b(\xi(t))$; then $\pi$ is faithful. Let $\left\{\delta_{s} \mid s \in G\right\}$ be the canonical basis of $l^{2}(G)$, and let $M_{|G|}$ be the matrix algebra with size $|G|$, where $\delta_{s}$ is the characterization function on $G$ of $\{s\} \subseteq G$. We have the map $\rho: M_{|G|} \rightarrow \mathbf{B}\left(l^{2}(G)\right)$, for $\left(x_{s t}\right) \in M_{|G|}$,

$$
\rho\left(\left(x_{s t}\right)\right)\left(\sum_{s \in G} \beta_{s} \delta_{s}\right)=\sum_{s \in G}\left(\sum_{t \in G} x_{s t} \beta_{t}\right) \delta_{s}, \quad \text { i.e. } \rho\left(\left(x_{s t}\right)\right)\left(\delta_{s}\right)=\sum_{t \in G} x_{t s} \delta_{t} .
$$

It is easy to see $\rho$ is an isomorphism, and $\pi \otimes \rho: B \otimes M_{|G|}=M_{|G|}(B) \rightarrow$ $\mathbf{B}\left(l^{2}(G, \mathbf{H})\right) \otimes \mathbf{B}\left(l^{2}(G)\right)=\mathbf{B}\left(l^{2}(G \times G, \mathbf{H})\right)$ is an injective homomorphism. By [12], 7.7.12, there is an injective homomorphism $\Phi: A \times_{\alpha} G \rightarrow \mathbf{B}\left(l^{2}(G, \mathbf{H})\right) \otimes \mathbf{B}\left(l^{2}(G)\right)=$ $\mathbf{B}\left(l^{2}(G \times G, \mathbf{H})\right)$ such that Image $(\Phi)=\operatorname{Image}(\pi \otimes \rho)$, and $\Phi$ is defined as follows: For every $b \in B, f, g \in C(G)$, and $Z(r, s)=b f(s-r) g(s) \in A \times{ }_{\alpha} G=C(G, B) \times{ }_{\alpha} G$, $\Phi(z)=\pi(b) \otimes v_{f g}$, where $v_{f g} \in \mathbf{B}\left(l^{2}(G)\right), v_{f g}(\eta)=\langle\eta, \bar{f}\rangle g, \forall \eta \in l^{2}(G)$. Therefore, we have isomorphism $\Psi=(\pi \otimes \rho)^{-1} \circ \Phi: A \times_{\alpha} G \rightarrow M_{|G|}(B)$, and so $R R\left(A \times_{\alpha} G\right)=R R(B)$.

Let $z_{t}=\delta_{e} \otimes \delta_{t} \otimes b \in C(G \times G, B) \subseteq A \times{ }_{\alpha} G$. Then $z_{t}(r, s)=b \delta_{e}(r) \delta_{t}(s)=$ $b \delta_{t}(s-r) \delta_{t}(s)$, and then $\Phi\left(z_{t}\right)=\pi(b) \otimes v_{\delta_{t} \delta_{t}}$. Let $v_{\delta_{t} \delta_{t}}=\rho\left(\left(x_{r s}\right)\right)$ with $\left(x_{r s}\right) \in$ $M_{|G|}$. Since $v_{\delta_{t} \delta_{t}}\left(\delta_{s}\right)=\left\langle\delta_{s}, \delta_{t}\right\rangle \delta_{t}=\delta_{s}(t) \delta_{t}$ and $\rho\left(\left(x_{r s}\right)\right)\left(\delta_{s}\right)=\sum_{r \in G} x_{r s} \delta_{r}, x_{r s}=$ $\delta_{r}(t) \delta_{s}(t)$. So $\left(x_{r s}\right)=E_{t t}$, i.e. $\rho\left(E_{t t}\right)=v_{\delta_{t} \delta_{t}}$, and $\Phi\left(z_{t}\right)=(\pi \otimes \rho)\left(b \otimes E_{t t}\right)$, where $E_{t t} \in M_{|G|}$ is the matrix with 1 at $(t, t)$ position, and 0 at other positions. Therefore $\Psi\left(z_{t}\right)=b \otimes E_{t t} \in B \otimes M_{|G|}=M_{|G|}(B)$. Let $\phi: T(B)=T\left(M_{|G|}(B)\right) \rightarrow T(A)_{\alpha^{*}}=$ $T(C(G) \otimes B)_{\alpha^{*}}$, for every $\tau \in T(B), \delta_{t} \otimes b \in C(G) \otimes B=A, \phi(\tau)\left(\delta_{t} \otimes b\right)=\frac{\tau(b)}{|G|}$. Then $\phi$ is an affine homeomorphism.

Let $\Psi^{*}$ be the affine map from $T(B)=T\left(M_{|G|}(B)\right)$ to $T\left(A \times_{\alpha} G\right)$ induced by $\Psi$. Then $\Psi^{*}$ is an affine homeomorphism, and for every $\tau \in T(B), \delta_{t} \otimes b \in A$,

$$
\left(R \circ \Psi^{*}\right)(\tau)\left(\delta_{t} \otimes b\right)=\Psi^{*}(\tau)\left(\delta_{e} \otimes \delta_{t} \otimes b\right)=\tau_{|G|}\left(\Psi\left(z_{t}\right)\right)=\tau_{|G|}\left(b \otimes E_{t t}\right)=\frac{\tau(b)}{|G|},
$$


where $\tau_{|G|} \in T\left(M_{|G|}(B)\right)$ is defined by $\tau \in T(B)$. So $R \circ \Psi^{*}=\phi$, i.e. we have the following commutative graph:

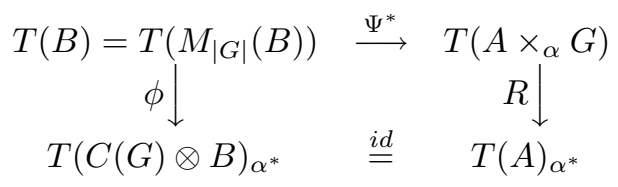

Therefore $R$ is an affine homeomorphism, but if $R R(B)$ is not 0 , then $R R\left(A \times{ }_{\alpha} G\right)=$ $R R(B)$ is also not 0 .

Proposition 2. Let $(A, G, \alpha)$ be a $C^{*}$-dynamical system with $A$ unital. For every $\phi \in \operatorname{Aff}\left(T(A)_{\alpha_{*}}\right), \psi \in \operatorname{AffT}(A)$, there are $a_{\phi}, a_{\psi} \in A_{\text {sa }}$, such that $\phi(\tau)=\tau\left(a_{\phi}\right)$ $\left(\forall \tau \in T(A)_{\alpha_{*}}\right), \psi(\tau)=\tau\left(a_{\psi}\right)(\forall \tau \in T(A))$. Therefore the restriction mapping $R_{G}$ from $\operatorname{AffT}(A)$ to $\operatorname{Aff}\left(T(A)_{\alpha_{*}}\right)$ is surjective.

Proof. Let $V, V_{\alpha}$ be the real linear subspace of $\left(A_{s a}\right)^{*}=\left(A^{*}\right)_{s a}$ which are linearly spanned by $T(A)$ and $T(A)_{\alpha_{*}}$ respectively; then $V_{\alpha} \subseteq V \subseteq\left(A_{s a}\right)^{*}$. Let

$$
\begin{aligned}
& W=\left\{\tau \in\left(A^{*}\right)_{s a}: \tau\left(u^{*} a u\right)=\tau(a), \forall a \in A, u \in U(A)\right\}, \\
& W_{\alpha}=\left\{\tau \in W: \tau\left(\alpha_{t}(a)\right)=\tau(a), \forall a \in A, t \in G\right\} .
\end{aligned}
$$

It is clear that $V \subseteq W, V_{\alpha} \subseteq W_{\alpha}$, and $W, W_{\alpha}$ are $w^{*}$-closed in $\left(A_{s a}\right)^{*}=\left(A^{*}\right)_{s a}$. Let $\tau \in W, \rho \in W_{\alpha}, \tau=\tau_{+}-\tau_{-}, \rho=\rho_{+}-\rho_{-}$be the Jordan decomposition of $\tau$ and $\rho$ respectively; then

$$
\tau_{+}, \tau_{-}, \rho_{+}, \rho_{-} \in A_{+}^{*},\|\tau\|=\left\|\tau_{+}\right\|+\left\|\tau_{-}\right\|,\|\rho\|=\left\|\rho_{+}\right\|+\left\|\rho_{-}\right\| .
$$

For any $u \in U(A), t \in G$, it is easy to see that

$$
\tau=\tau_{+}\left(u^{*} \cdot u\right)-\tau_{-}\left(u^{*} \cdot u\right), \rho=\rho_{+}\left(u^{*} \cdot u\right)-\rho_{-}\left(u^{*} \cdot u\right),
$$

and

$$
\rho=\rho_{+}\left(\alpha_{t}(\cdot)\right)-\rho_{-}\left(\alpha_{t}(\cdot)\right) .
$$

Since $\left\|\tau_{+}\right\|=\left\|\tau_{+}\left(u^{*} \cdot u\right)\right\|, \quad\left\|\tau_{-}\right\|=\left\|\tau_{-}\left(u^{*} \cdot u\right)\right\|, \quad\left\|\rho_{+}\right\|=\left\|\rho_{+}\left(u^{*} \cdot u\right)\right\|, \quad\left\|\rho_{-}\right\|=$ $\left\|\rho_{-}\left(u^{*} \cdot u\right)\right\|$, and $\left\|\rho_{+}\left(\alpha_{t}(\cdot)\right)\right\|=\left\|\rho_{+}\right\|,\left\|\rho_{-}\right\|=\left\|\rho_{-}\left(\alpha_{t}(\cdot)\right)\right\|$, by the uniqueness of the Jordan decomposition, $\tau_{+}=\tau_{+}\left(u^{*} \cdot u\right), \tau_{-}=\tau_{-}\left(u^{*} \cdot u\right), \rho_{+}=\rho_{+}\left(u^{*} \cdot u\right), \rho_{-}=\rho_{-}\left(u^{*} \cdot u\right)$, $\rho_{+}=\rho_{+} \circ \alpha_{t}, \rho_{-}=\rho_{-} \circ \alpha_{t}$. Therefore

$$
\frac{\tau_{+}}{\left\|\tau_{+}\right\|}, \frac{\tau_{-}}{\left\|\tau_{-}\right\|} \in T(A), \quad \frac{\rho_{+}}{\left\|\rho_{+}\right\|}, \frac{\rho_{-}}{\left\|\rho_{-}\right\|} \in T(A)_{\alpha_{*}},
$$

and so

$$
\tau=\left\|\tau_{+}\right\| \frac{\tau_{+}}{\left\|\tau_{+}\right\|}-\left\|\tau_{-}\right\| \frac{\tau_{-}}{\left\|\tau_{-}\right\|} \in V, \quad \rho=\left\|\rho_{+}\right\| \frac{\rho_{+}}{\left\|\rho_{+}\right\|}-\left\|\rho_{-}\right\| \frac{\rho_{-}}{\left\|\rho_{-}\right\|} \in V_{\alpha},
$$

i.e. $W \subseteq V$ and $W_{\alpha} \subseteq V_{\alpha}$. Therefore $W=V$ and $W_{\alpha}=V_{\alpha}$; as a consequence, $V$ and $V_{\alpha}$ are $w^{*}$-closed in $\left(A^{*}\right)_{s a}=\left(A_{s a}\right)^{*}$.

Let $\phi \in \operatorname{Aff}\left(T(A)_{\alpha_{*}}\right)$, and we define $g$ to be the linear functional on $V_{\alpha}: g(\tau)=$ $\lambda \phi\left(\tau_{1}\right)-\mu \phi\left(\tau_{2}\right)$, if $\tau=\lambda \tau_{1}-\mu \tau_{2} \in V_{\alpha}$ with $\lambda, \mu \in \mathbf{R}_{+} \cup\{0\}, \tau_{1}, \tau_{2} \in T(A)_{\alpha_{*}}$. If $\tau=\lambda^{\prime} \tau_{1}^{\prime}-\mu^{\prime} \tau_{2}^{\prime}$ is another decomposition with $\lambda^{\prime}, \mu^{\prime} \in \mathbf{R}_{+} \cup\{0\}, \tau_{1}^{\prime}, \tau_{2}^{\prime} \in T(A)_{\alpha_{*}}$, then $\lambda \tau_{1}+\mu^{\prime} \tau_{2}^{\prime}=\lambda^{\prime} \tau_{1}^{\prime}+\mu \tau_{2}$. Since $\lambda^{\prime}+\mu=\lambda^{\prime} \tau_{1}^{\prime}(1)+\mu \tau_{2}(1)=\lambda \tau_{1}(1)+\mu^{\prime} \tau_{2}^{\prime}(1)=\lambda+\mu^{\prime}$, $\frac{\lambda^{\prime}}{\lambda^{\prime}+\mu} \tau_{1}^{\prime}+\frac{\mu}{\lambda^{\prime}+\mu} \tau_{2}=\frac{\lambda}{\lambda+\mu^{\prime}} \tau_{1}+\frac{\mu^{\prime}}{\lambda+\mu^{\prime}} \tau_{2}^{\prime} \in T(A)_{\alpha_{*}}$. So

$$
\frac{\lambda^{\prime}}{\lambda^{\prime}+\mu} \phi\left(\tau_{1}^{\prime}\right)+\frac{\mu}{\lambda^{\prime}+\mu} \phi\left(\tau_{2}\right)=\frac{\lambda}{\lambda+\mu^{\prime}} \phi\left(\tau_{1}\right)+\frac{\mu^{\prime}}{\lambda+\mu^{\prime}} \phi\left(\tau_{2}^{\prime}\right) .
$$


Therefore $\lambda \phi\left(\tau_{1}\right)-\mu \phi\left(\tau_{2}\right)=\lambda^{\prime} \phi\left(\tau_{1}^{\prime}\right)-\mu^{\prime} \phi\left(\tau_{2}^{\prime}\right)$, and so the definition of $g$ is well defined. It is also easy to see $g$ is linear on $V_{\alpha}$. Let $\left\{\tau_{i}\right\}_{i \in \Lambda}$ be a bounded net in $V_{\alpha}$ with $\tau_{i}$ $w^{*}$-convergent to $\tau$ in $V_{\alpha}$. If $g\left(\tau_{i}\right)$ is not convergent to $g(\tau)$, then there is a $\varepsilon>0$ such that $\left\{i \in \Lambda:\left|g\left(\tau_{i}\right)-g(\tau)\right| \geq \varepsilon\right\}=s_{\Lambda}$ is a directed subset of $\Lambda$ with the order of $\Lambda$. So $\left\{\tau_{j}\right\}_{j \in s_{\Lambda}}$ is a subnet of $\left\{\tau_{i}\right\}_{i \in \Lambda}$.

Let $\tau_{i}=\left(\tau_{i}\right)_{+}-\left(\tau_{i}\right)_{-}$be the Jordan decomposition of $\tau_{i}$; then

$$
\left\|\tau_{i}\right\|=\left\|\left(\tau_{i}\right)_{+}\right\|+\left\|\left(\tau_{i}\right)_{-}\right\| .
$$

Since $\left\{\left\|\tau_{i}\right\|, i \in \Lambda\right\}$ is bounded, both $\left\{\left\|\left(\tau_{i}\right)_{+}\right\|, i \in \Lambda\right\}$ and $\left\{\left\|\left(\tau_{i}\right)_{-}\right\|, i \in \Lambda\right\}$ are bounded, too. Since the bounded closed ball of $\left(A_{s a}\right)^{*}$ is $w^{*}$ compact, there are subnets $\left\{\left(\tau_{k}\right)_{+}\right\} \subseteq\left\{\left(\tau_{j}\right)_{+}: j \in s_{\Lambda}\right\}$ and $\left\{\left(\tau_{k}\right)_{-}\right\} \subseteq\left\{\left(\tau_{j}\right)_{-}: j \in s_{\Lambda}\right\}$ with $\left(\tau_{k}\right)_{+}$ and $\left(\tau_{k}\right)_{-} w^{*}$ convergent to $\rho_{1}$ and $\rho_{2}$ in $V_{\alpha}$, respectively. So $\tau=\rho_{1}-\rho_{2}$, and $\left(\tau_{k}\right)_{+}(1) \rightarrow \rho_{1}(1),\left(\tau_{k}\right)_{-}(1) \rightarrow \rho_{2}(1)$. Therefore $\frac{\left(\tau_{k}\right)_{+}}{\left(\tau_{k}\right)_{+}(1)} \in T(A)_{\alpha_{*}}$ is $w^{*}$-convergent to $\frac{\rho_{1}}{\rho_{1}(1)} \in T(A)_{\alpha_{*}}$, and $\frac{\left(\tau_{k}\right)_{-}}{\left(\tau_{k}\right)_{-}(1)} \in T(A)_{\alpha_{*}}$ is $w^{*}$-convergent to $\frac{\rho_{2}}{\rho_{2}(1)} \in T(A)_{\alpha_{*}}$ (here, without loss of generality, we assume that all the denominators are not zero). Then

$$
\begin{aligned}
& g\left(\tau_{k}\right)=\left(\tau_{k}\right)_{+}(1) \phi\left(\frac{\left(\tau_{k}\right)_{+}}{\left(\tau_{k}\right)_{+}(1)}\right)-\left(\tau_{k}\right)_{-}(1) \phi\left(\frac{\left(\tau_{k}\right)_{-}}{\left(\tau_{k}\right)_{-}(1)}\right) \\
& \longmapsto \rho_{1}(1) \phi\left(\frac{\rho_{1}}{\rho_{1}(1)}\right)-\rho_{2}(1) \phi\left(\frac{\rho_{2}}{\rho_{2}(1)}\right)=g\left(\rho_{1}-\rho_{2}\right)=g(\tau),
\end{aligned}
$$

i.e.

$$
g\left(\tau_{k}\right) \rightarrow g(\tau) .
$$

This contradicts the fact that $\left|g\left(\tau_{k}\right)-g(\tau)\right| \geq \varepsilon$, and so $g\left(\tau_{i}\right) \rightarrow g(\tau)$. By the KreinSmulian Theorem, we see that $g$ is a $w^{*}$-continuous linear functional on $V_{\alpha}$ with $\left.g\right|_{T(A)_{\alpha_{*}}}=\phi$. By the Hahn-Banach extension theorem, there is a $w^{*}$-continuous linear functional on $\left(A_{s a}\right)^{*}$ which is an extension of $g$. By the well-known dual theorem, there is an element $a_{\phi} \in A_{s a}$ such that $g(\tau)=\tau\left(a_{\phi}\right)\left(\forall \tau \in V_{\alpha}\right)$, and so $\phi(\tau)=\tau\left(a_{\phi}\right)\left(\forall \tau \in T(A)_{\alpha_{*}}\right)$.

Similar discussion says, for any $\psi \in \operatorname{AffT}(A)$, there is also $a_{\psi} \in A_{s a}$ such that $\psi(\tau)=\tau\left(a_{\psi}\right)(\forall \tau \in T(A))$.

Lemma 3 ([16], Theorem 3.2). For $n \in \mathbf{N} \cup\{\infty\}, \Delta_{n}$ induces a homeomorphic group isomorphism

$$
\Delta_{n}: U_{0}^{n}(A) / \overline{D U_{0}^{n}(A)} \mapsto \operatorname{Aff} T(A) / \overline{{\Delta_{n}^{0}\left(\pi_{1}\left(U_{0}^{n}(A)\right)\right)}}
$$

with $\Phi_{n}=\Delta_{n}^{-1}$ defined as follows: By the duality theorem, for any element $\xi$ in Aff $T(A)$, we have $a \in A_{\text {sa }}$ with $\xi(\tau)=\tau(a)$ for any $\tau \in T(A)$, and so denote $\xi$ by $\hat{a}$. Then define $\Phi_{n}(q(\xi))=q^{0}\left(e^{2 \pi i a}\right)$. In particular,

$$
U_{0}^{\infty}(A) / \overline{D U_{0}^{\infty}(A)} \cong \operatorname{AffT}(A) / \overline{\rho\left(K_{0}(A)\right)} .
$$

Lemma 4. Let $(A, G, \alpha)$ be a $C^{*}$-dynamical system with $A$ unital and $G$ abelian and discrete, let $R_{G}$ be the restriction map from $\operatorname{Aff} T(A)$ to $\operatorname{Aff}\left(T(A)_{\alpha_{*}}\right)$, let $R^{*}$ be the homomorphism from Aff $\left(T(A)_{\alpha_{*}}\right)$ to AffT $\left(A \times_{\alpha} G\right)$ induced by the affine map $R: T\left(A \times_{\alpha} G\right) \rightarrow T(A)_{\alpha_{*}}$ defined in Lemma 2 , and let $\Psi=R^{*} \circ R_{G}$. Then $\Psi: \operatorname{AffT}(A) \rightarrow \operatorname{AffT}\left(A \times_{\alpha} G\right)$ maps $\overline{\Delta_{n}^{0}\left(\pi_{1}\left(U_{0}^{n}(A)\right)\right)}$ into $\overline{\Delta_{n}^{0}\left(\pi_{1}\left(U_{0}^{n}\left(A \times_{\alpha} G\right)\right)\right)}$, and induces a group homomorphism from AffT $(A) \sqrt{\Delta_{n}^{0}\left(\pi_{1}\left(U_{0}^{n}(A)\right)\right)}$ to 
$\operatorname{AffT}\left(A \times_{\alpha} G\right) \overline{\Delta_{n}^{0}\left(\pi_{1}\left(U_{0}^{n}\left(A \times_{\alpha} G\right)\right)\right)}$, which is denoted by $\psi$, such that the diagram

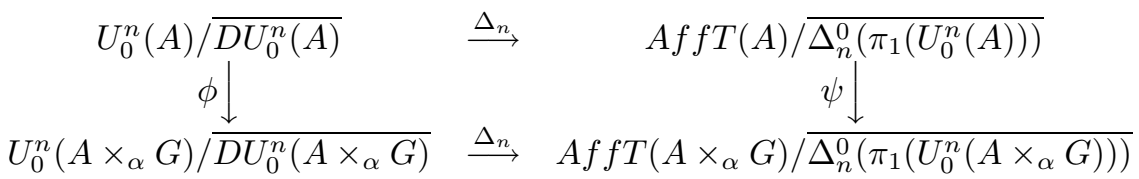

commutes, where $\phi$ is induced by the inclusion map from $U_{0}^{n}(A)$ to $U_{0}^{n}\left(A \times_{\alpha} G\right)$.

Proof. Let $\gamma$ be a closed piecewise smooth path based at the identity of $U_{0}^{n}(A)$, and let $[\gamma]_{A}$ be the element in $\pi_{1}\left(U_{0}^{n}(A)\right)$ with representative $\gamma$. Then $\gamma$ can also be viewed as a closed piecewise smooth path in $U_{0}^{n}\left(A \times_{\alpha} G\right)$, and let $[\gamma]_{A \times_{\alpha} G}$ be the element in $\pi_{1}\left(U_{0}^{n}\left(A \times{ }_{\alpha} G\right)\right)$ with representative $\gamma$. Let $\omega \in T\left(A \times_{\alpha} G\right)$; then

$$
\begin{aligned}
& \Psi\left(\Delta_{n}^{0}\left([\gamma]_{A}\right)\right)(\omega)=R_{G}\left(\Delta_{n}^{0}\left([\gamma]_{A}\right)\right)(R(\omega)) \\
& =\frac{1}{2 \pi i} \int_{0}^{1} R(\omega)\left(\gamma^{\prime}(t) \gamma(t)^{*}\right) d t=\Delta_{n}^{0}\left([\gamma]_{A \times_{\alpha} G}\right)(\omega) .
\end{aligned}
$$

So $\Psi\left(\Delta_{n}^{0}\left([\gamma]_{A}\right)\right)=\Delta_{n}^{0}\left([\gamma]_{A \times{ }_{\alpha} G}\right)$. Since $R^{*}$ and $R_{G}$ are continuous in norm topology, so is $\Psi$. Therefore $\Psi$ maps $\overline{\Delta_{n}^{0}\left(\pi_{1}\left(U_{0}^{n}(A)\right)\right)}$ into $\overline{\Delta_{n}^{0}\left(\pi_{1}\left(U_{0}^{n}\left(A \times_{\alpha} G\right)\right)\right)}$. Let $a \in A_{s a}$, $\hat{a} \in \operatorname{AffT}(A), \hat{a}(\tau)=\tau(a), \forall \tau \in T(A)$. Then $\Delta_{n}^{-1}(q(\hat{a}))=q^{0}\left(e^{2 \pi i a}\right)$, and so $\phi \circ \Delta_{n}^{-1}(q(\hat{a}))=q_{G}^{0}\left(e^{2 \pi i a}\right)$, where $q_{G}^{0}$ is the quotient map from $U_{0}^{n}\left(A \times_{\alpha} G\right)$ to $U_{0}^{n}\left(A \times_{\alpha} G\right) / \overline{D U_{0}^{n}\left(A \times_{\alpha} G\right)}$. Since for any $\omega \in T\left(A \times_{\alpha} G\right)$,

$$
\Psi(\hat{a})(\omega)=R_{G}(\hat{a})(R(\omega))=R(\omega)(a)=\omega(a)=\hat{a}(\omega),
$$

$\psi(q(\hat{a}))=q_{G}(\Psi(\hat{a}))=q_{G}(\hat{a})$, where $q_{G}$ is the quotient map from $\operatorname{AffT}\left(A \times_{\alpha} G\right)$ to $\operatorname{Aff} T\left(A \times_{\alpha} G\right) / \overline{\Delta_{n}^{0}\left(\pi_{1}\left(U_{0}^{n}\left(A \times_{\alpha} G\right)\right)\right)}$. Therefore $\left(\Delta_{n}^{-1} \circ \psi\right)(q(\hat{a}))=\Delta_{n}^{-1}\left(q_{G}(\hat{a})\right)=$ $q_{G}^{0}\left(e^{2 \pi i a}\right)=\phi \circ \Delta_{n}^{-1}(q(\hat{a}))$, i.e. $\phi \circ \Delta_{n}^{-1}=\Delta_{n}^{-1} \circ \psi$.

Theorem 2. Let $(A, G, \alpha)$ be a $C^{*}$-dynamical system with $A$ unital, $G$ abelian and discrete, and with $R: T\left(A \times \times_{\alpha} G\right) \rightarrow T(A)_{\alpha_{*}}$ homeomorphic. Then $A \times_{\alpha} G$ is of real rank zero if and only if each unitary element in $A \times_{\alpha} G$ with the form $u_{A} \prod_{i=1}^{n} x_{i}^{*} y_{i}^{*} x_{i} y_{i}$ can be approximated by the unitary elements in $A \times_{\alpha} G$ with finite spectrum, where $u_{A} \in U_{0}(A), x_{i}, y_{i} \in C_{c}(G, A) \cap U_{0}\left(A \times_{\alpha} G\right)$.

Proof. Keeping the notations as above, by Proposition 2, $R_{G}: \operatorname{AffT}(A) \rightarrow$ $\operatorname{Aff}\left(T(A)_{\alpha^{*}}\right)$ is surjective. Since $R$ is homeomorphic, $\Psi$ is surjective, and so is $\psi$. Then $\phi: U_{0}(A) / \overline{D U_{0}(A)} \rightarrow U_{0}\left(A \times_{\alpha} G\right) / \overline{D U_{0}\left(A \times_{\alpha} G\right)}$ is surjective by Lemma 4. For any $u \in U_{0}\left(A \times_{\alpha} G\right)$, there exists $u_{A} \in U_{0}(A)$ such that $\phi\left(q^{0}\left(u_{A}\right)\right)=q_{G}^{0}(u)$, and so there exists $v \in \overline{D U_{0}\left(A \times_{\alpha} G\right)}$ such that $u=u_{A} v$. By definition, $v$ can be approximated by the unitary elements with the form $\prod_{i=1}^{n} \bar{x}_{i}^{*} \bar{y}_{i}^{*} \bar{x}_{i} \bar{y}_{i}$, where $\bar{x}_{i}$, $\bar{y}_{i} \in U_{0}\left(A \times{ }_{\alpha} G\right)$. For arbitrary $\bar{x} \in U_{0}\left(A \times{ }_{\alpha} G\right)$, it is well known that $\bar{x}$ can be approximated by the unitary elements with the form $e^{i h_{1}} e^{i h_{2}} \cdots e^{i h_{k}}$ for some $h_{1}, h_{2}, \cdots, h_{k} \in\left(A \times{ }_{\alpha} G\right)_{s a}$. Since for each $i(1 \leq i \leq k), h_{i}$ can be approximated by the self-adjoint elements in $C_{c}(G, A) \subseteq A \times_{\alpha} G, \bar{x}$ can be approximated by the elements in $C_{c}(G, A) \cap U_{0}\left(A \times_{\alpha} G\right)$, and so we can choose the $\bar{x}_{i}$ and $\bar{y}_{i}$ above in $C_{c}(G, A) \cap U_{0}\left(A \times_{\alpha} G\right)$. Then the remainder of the proof is from [11, 4.2.8]

Proposition 3. Let $A$ be a unital inductive limit of the direct sums of nonelementary simple $C^{*}$-algebras of real rank zero. Then $U_{0}^{n}(A)=\overline{D U_{0}^{n}(A)}$. 
Proof. By Lemma 3, it is enough to prove that $\operatorname{AffT}(A)=\overline{\Delta_{1}^{0}\left(\pi_{1}\left(U_{0}(A)\right)\right)}$. Let $A=\lim _{n \rightarrow \infty}\left(A_{n}, \phi_{n}\right)$, where $A_{n}=\bigoplus_{i=1}^{k_{n}} A_{n, i}$, and all $A_{n, i}$ are non-elementary simple $C^{*}$-algebras of real rank zero. Since $A_{n, i}$ is simple, we may assume all $\phi_{n}: A_{n} \rightarrow A$ are injective and view $A_{n}$ as a subalgebra of $A$. Let $a \in\left(A_{n}\right)_{s a}$; then $a=\bigoplus_{i=1}^{k_{n}} a_{i}$ and $\hat{a}=\bigoplus_{i=1}^{k_{n}} \hat{a_{i}} \in \operatorname{AffT}(A)$. Since $A_{n, i}$ is of real rank zero, $\hat{a_{i}} \in \overline{\operatorname{span}} \mathbf{R}_{\mathbf{p}}\{\hat{p}: p=$ $\left.p^{*}=p^{2} \in A_{n, i}\right\}$.

Let $p \in A_{n, i}$ be a projection, and $r \in \mathbf{R}$. Then for any $\varepsilon>0$, we can choose $n, m \in \mathbf{N}$ such that $\left|r-\frac{m}{2^{n}}\right|<\varepsilon,\left|\frac{r}{2^{n}}\right|<\varepsilon$. By [18, Theorem 2.1], there are equivalent orthogonal subprojections $p_{1}, p_{2}, \cdots, p_{2^{n}}$ of $p$ and subprojection $q$ of $p$ such that $p \sim \sum_{i=1}^{2^{n}} p_{i} \oplus q$ and $q \preceq p_{1}$, and so $\hat{p}=2^{n} \hat{p}_{1}+\hat{q}, \hat{q} \leq \hat{p}_{1}$. Then

$$
\begin{aligned}
& \left\|r \hat{p}-m \hat{p}_{1}\right\| \leq\left\|r \hat{p}-\frac{m}{2^{n}} \hat{p}\right\|+\left\|\frac{m}{2^{n}} \hat{p}-m \hat{p}_{1}\right\| \\
& \leq \varepsilon+\frac{m\|\hat{q}\|}{2^{n}} \leq \varepsilon+\frac{m\left\|\hat{p}_{1}\right\|}{2^{n}} \leq \varepsilon+\frac{m}{2^{n}} \frac{\|\hat{p}\|}{2^{n}} \\
& \leq 2 \varepsilon+\frac{r}{2^{n}} \leq 3 \varepsilon .
\end{aligned}
$$

Therefore $\overline{\operatorname{span}}_{\mathbf{R}}\left\{\hat{p}: p=p^{*}=p^{2} \in A_{n, i}\right\}=\overline{\operatorname{span}}_{\mathbf{Z}}\left\{\hat{p}: p=p^{*}=p^{2} \in A_{n, i}\right\}$.

By the discussion above, $\hat{a} \in \overline{\operatorname{span}}_{\mathbf{Z}}\left\{\hat{p}: p=p^{*}=p^{2} \in A_{n}\right\} \subseteq \overline{\operatorname{span}}_{\mathbf{Z}}\{\hat{p}: p=$ $\left.p^{*}=p^{2} \in A\right\}$. For a projection $p$ in $A$, we have $\eta:[0,1] \rightarrow U_{0}(A), \eta(t)=e^{2 \pi i t p}$. It is easy to see that $\Delta_{1}^{1}(\eta)=\hat{p}$, so $\hat{p} \in \Delta_{1}^{0}\left(\pi_{1}\left(U_{0}(A)\right)\right)$. Therefore $\hat{a} \in \overline{\operatorname{span}}_{\mathbf{Z}}\{\hat{p}$ : $\left.p=p^{*}=p^{2} \in A\right\} \subseteq \overline{\Delta_{1}^{0}\left(\pi_{1}\left(U_{0}(A)\right)\right)}$. This completes the proof of AffT $(A)=$ $\overline{\Delta_{1}^{0}\left(\pi_{1}\left(U_{0}(A)\right)\right)}$.

Note. The assumption is necessary that the direct sums of the building blocks are non-elementary. For example let $A=M_{n}(\mathbf{C})(n \geq 1)$. Then $U_{0}^{n}(A)=U^{n}(A)$ and $\overline{D U^{n}(A)} \neq U_{0}^{n}(A)$, since for any $a \in \overline{D U^{n}(A)}$, the determinant $|a|$ of $a$ must be 1 .

Theorem 3. Let $A$ be a unital inductive limit of the direct sums of non-elementary simple $C^{*}$-algebras of real rank zero, let $(A, G, \alpha)$ be a $C^{*}$-dynamical system with $G$ abelian and discrete, and let $R: T\left(A \times_{\alpha} G\right) \rightarrow T(A)_{\alpha_{*}}$ be homeomorphic. Then

(1) $U_{0}^{n}\left(A \times_{\alpha} G\right)=\overline{D U_{0}^{n}\left(A \times_{\alpha} G\right)}$.

(2) $A \times{ }_{\alpha} G$ is of real rank zero if and only if each unitary element in $A \times{ }_{\alpha} G$ with the form $\prod_{i=1}^{n} x_{i}^{*} y_{i}^{*} x_{i} y_{i}$ can be approximated by the unitary elements in $A \times_{\alpha} G$ with finite spectrum, where $x_{i}, y_{i} \in C_{c}(G, A) \cap U_{0}\left(A \times_{\alpha} G\right)$.

Proof. From the discussion in the proof of Theorem 2, we know that $\phi$ defined in Lemma 4 is surjective. Then (1) is from Lemma 4 and Proposition 3 directly. The proof of (2) is similar to that of Theorem 2 by use of (1).

\section{ACKNOWLEDGEMENTS}

The author is grateful to the referee for his many valuable suggestions such that the presentation of the paper has become much more readable.

\section{REFERENCES}

[1] De la Harpe, P. and Skandalis, G., Dèterminant Associè á une Trace sur une Algebrè de Banach, Ann. Inst. Fourier (Grenoble), 34-1(1984), 241-260. MR0743629 (87i:46146a)

[2] Blackadar, B., K-theory for Operator Algebras, Springer-Verlag, New York, 1986. MR0859867 (88g:46082)

[3] Bratteli, O., Evans, D. E. and Kishimoto, A., The Rokhlin Property for Quasi-Free Automomorphism of the Fermion Algebra, Proc. London Math. Soc., 71(1995), 675-694. MR1347409 (97g:46083) 
[4] Brown, P. L. and Pedersen, G. K., $C^{*}$-algebras of Real Rank Zero, J. Funct. Anal. 99(1991), 131-149. MR.1120918 (92m:46086)

[5] Elliott, G. A., On the Classification of $C^{*}$-algebras of Real Rank Zero, J. Reine Angew. Math., 443(1993), 179-219. MR1241132 (94i:46074)

[6] Elliott, G. A., Gong, G. and Li, L., On the Classification of Simple Inductive Limit C* algebras, II: The Isomorphism Theorem, preprint.

[7] Elliott, G. A. and Fang, X., Simple Inductive Limits of $C^{*}$-algebras with Building Blocks from Spheres of Odd Dimension, Contemp. Math.(228), 'Operator Algebra and Operator Theory', 1998, 79-86. MR.1667655 (2000k:46077)

[8] Fang, X., The Simplicity and Real Rank Zero Property of the Inductive Limit of Continuous Trace $C^{*}$-algebras, Analysis, 19 (1999), 377-389. MR1743530 (2001k:46085)

[9] Kishimoto, A., The Rokhlin Property for Automorphism of the UHF Algebras, J. Reine Angew. Math., 465(1995), 183-196. MR.1344136(96k:46114)

[10] Lin, H., Almost Multiplicative Morphisms and Some Applications, J. Operator Theory 37(1997), 121-154. MR1438204 (98b:46091)

[11] Lin, H., An Introduction to the Classification of Amenable $C^{*}$-algebras, World Scientific, Singapore, 2001. MR 1884366 (2002k:46141)

[12] Pedersen, G. K., $C^{*}$-algebras and Their Automorphism Groups, Academic Press, London and New York, 1979. MR0548006 (81e:46037)

[13] Rieffel, M. Dimension and Stable Rank in the K-theory of $C^{*}$-algebras, Proc. London Math. Soc., 46(1983), 301-333. MR0693043 (84g:46085)

[14] Rördam, M. Classification of Inductive Limits of Cuntz Algebras, J. Reine Angew. Math., 440(1993), 175-200. MR1225963 (94k:46120)

[15] Thomsen, K., Diagonalization in Inductive Limits of Circle Algebras, J. Operator Theory, 27(1992), 325-340. MR.1249649 (95f:46098)

[16] Thomsen, K., Trace, Unitary Characters and Crossed Products by Z, Publ. RIMS. Kyoto Univ., 31(1995), 1011-1029. MR.1382564 (97a:46074)

[17] Villadsen, J., Simple $C^{*}$-algebras with Perforation, J. Funct. Anal. 154(1998), 110-116. MR.1616504 (99j:46069)

[18] Zhang, S., Matricial Structure and Homotopy Type of Simple $C^{*}$-algebras with Real Rank Zero, J. Operator Theory 26(1991), 283-312. MR1225518 (94f:46075)

[19] Zhang, S., On the Homotopy Type of the Unitary Group and the Grassmann Space of Purely Infinite Simple $C^{*}$-algebras, K-Theory 24(2001), 203-225. MR1876798 (2002m:46088)

Department of Applied Mathematics, Tongui University, Shanghai, 200092, People's Republic of China

E-mail address: xfang@mail.tongji.edu.cn 